\title{
Highly Homogeneous Biotinylated Carbon Nanodots: Red-emitting Nano-heaters as Theranostic Agents Towards Precision Cancer Medicine
}

Cinzia Scialabba, ${ }^{\mathrm{a}}$ Alice Sciortino, ${ }^{\mathrm{b}}$ Fabrizio Messina, ${ }^{\mathrm{b}}$ Gianpiero Buscarino, ${ }^{\mathrm{b}}$ Marco Cannas, ${ }^{\mathrm{b}}$ Giuseppina Roscigno, ${ }^{\text {cd }}$ Gerolama Condorelli, ${ }^{\mathrm{c}}$ Gennara Cavallaro, ${ }^{\mathrm{a}}$ Gaetano Giammona ${ }^{\mathrm{a}}$ and Nicolò Mauro $^{\text {ad* }}$

a Laboratory of Biocompatible Polymers, Department of "Scienze e Tecnologie Biologiche, Chimiche e Farmaceutiche" (STEBICEF), University of Palermo, Via Archirafi, 32, 90123 Palermo, Italy.

b Dipartimento di Fisica e Chimica, Università Degli Studi di Palermo, Via Archirafi 36, 90123 Palermo, Italy

c Department of Molecular Medicine and Medical Biotechnology, "Federico II" University of Naples, Naples, Italy

${ }^{\mathrm{d}}$ Fondazione Umberto Veronesi, Piazza Velasca 5, 20122 Milano, Italy

Corresponding Author

*E-mail: nicolo.mauro@unipa.it. Fax: +39 09123891928. Tel.: +39 09123891928

\section{ORCID}

Nicolò Mauro: 000-0003-0246-3474 


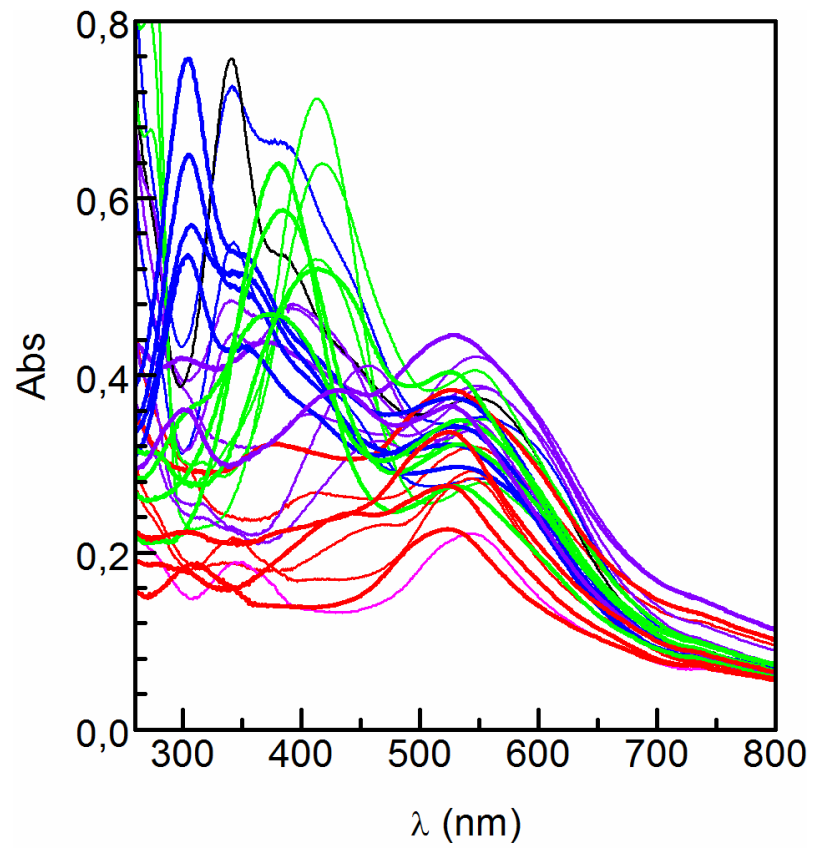

Figure S1. UV absorption spectra of the CDs fractions retrieved during SEC purification of crude CDs. Curves are colored on the basis of the typical emission observed: from blue to red emission.
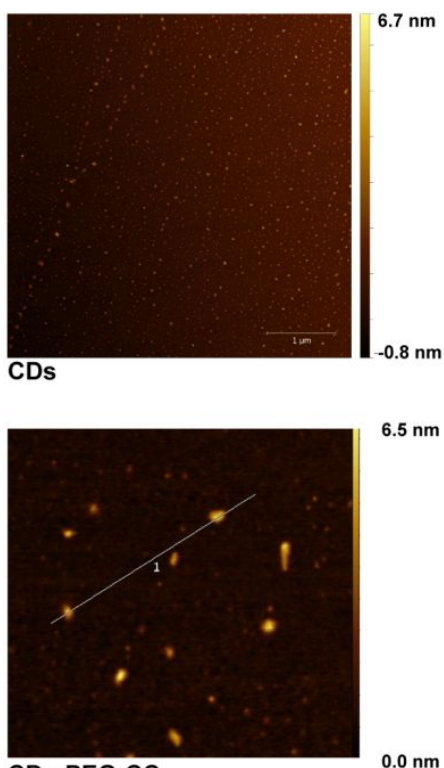

$6.5 \mathrm{~nm}$

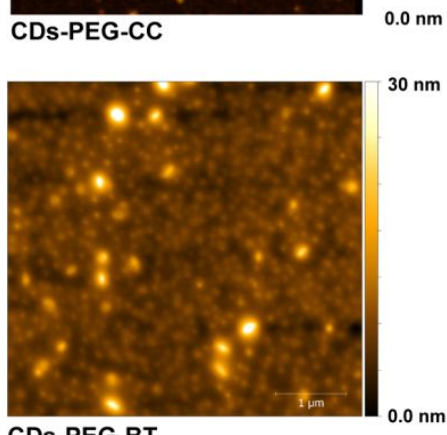

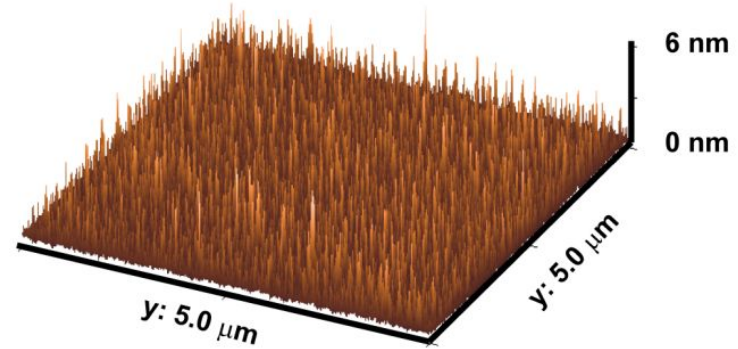
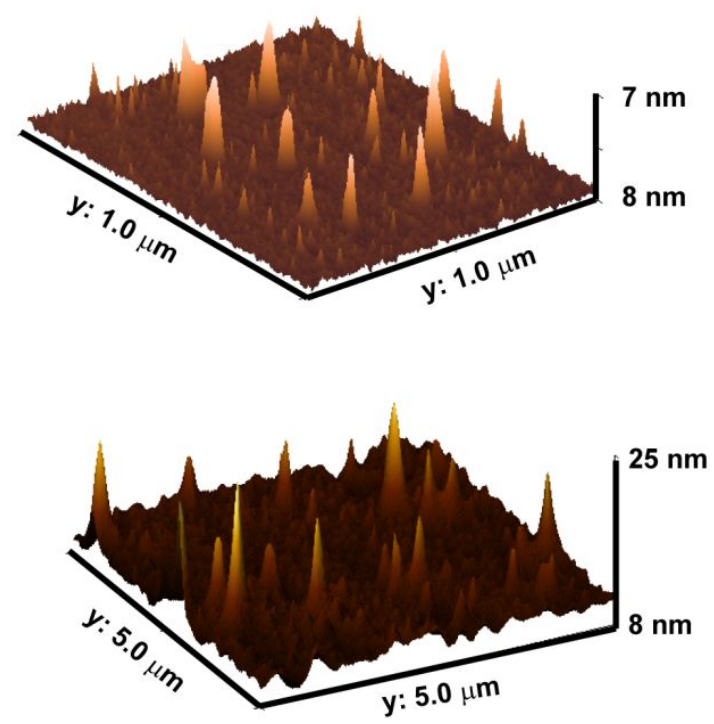
Figure S2. Atomic force microscopy (AFM) of the selected CDs and their derivatives

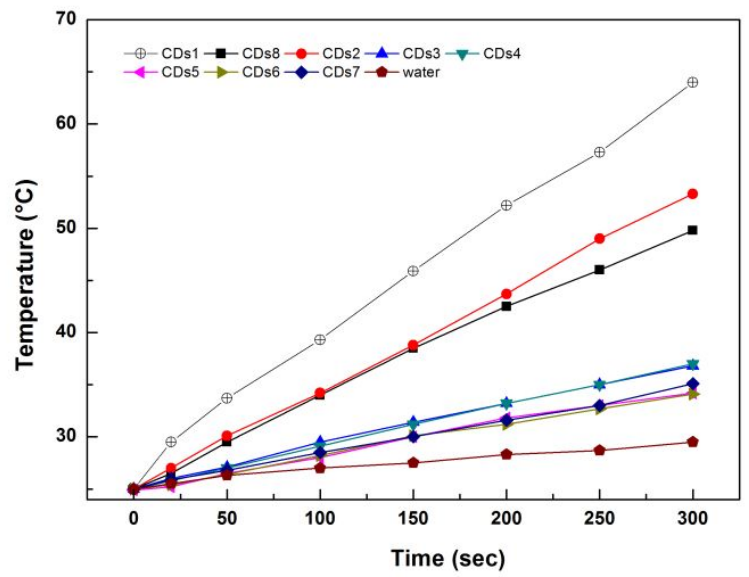

Figure S3. Photothermal effect of the CDs fractions obtained after SEC purification $\left(0.1 \mathrm{mg} \mathrm{mL}^{-1}\right)$
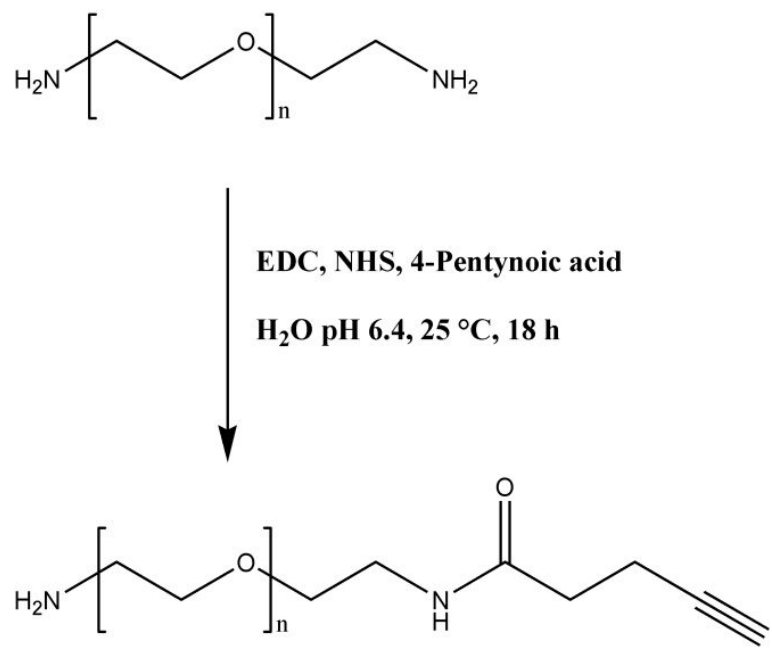

Figure S4. Schematic representation of the synthesis of the amino-PEG-alkyne 


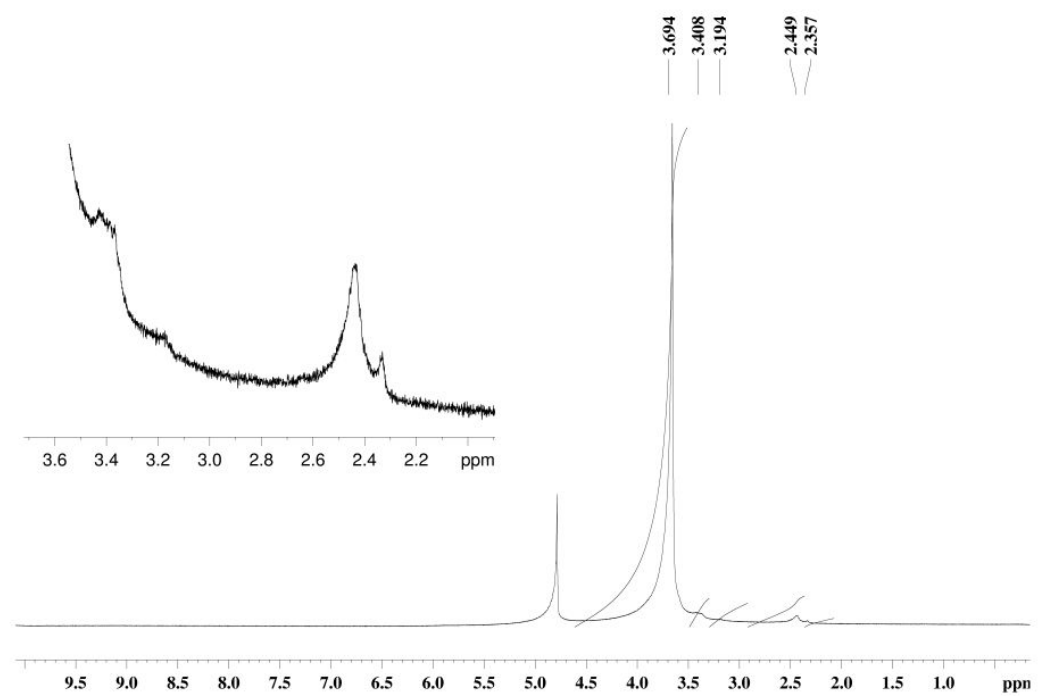

a)

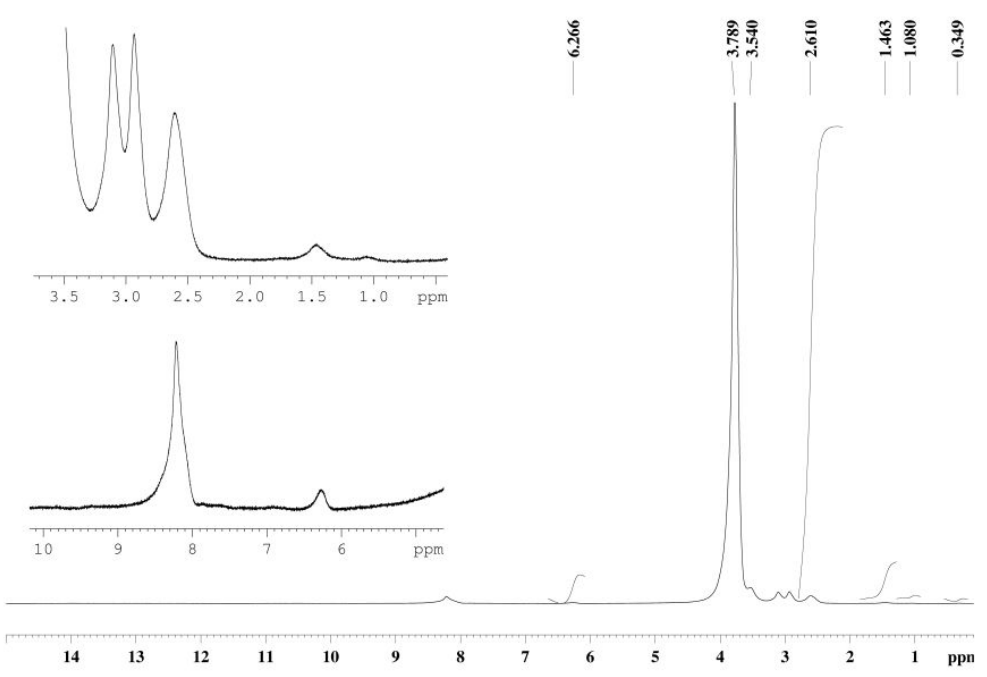

b) 


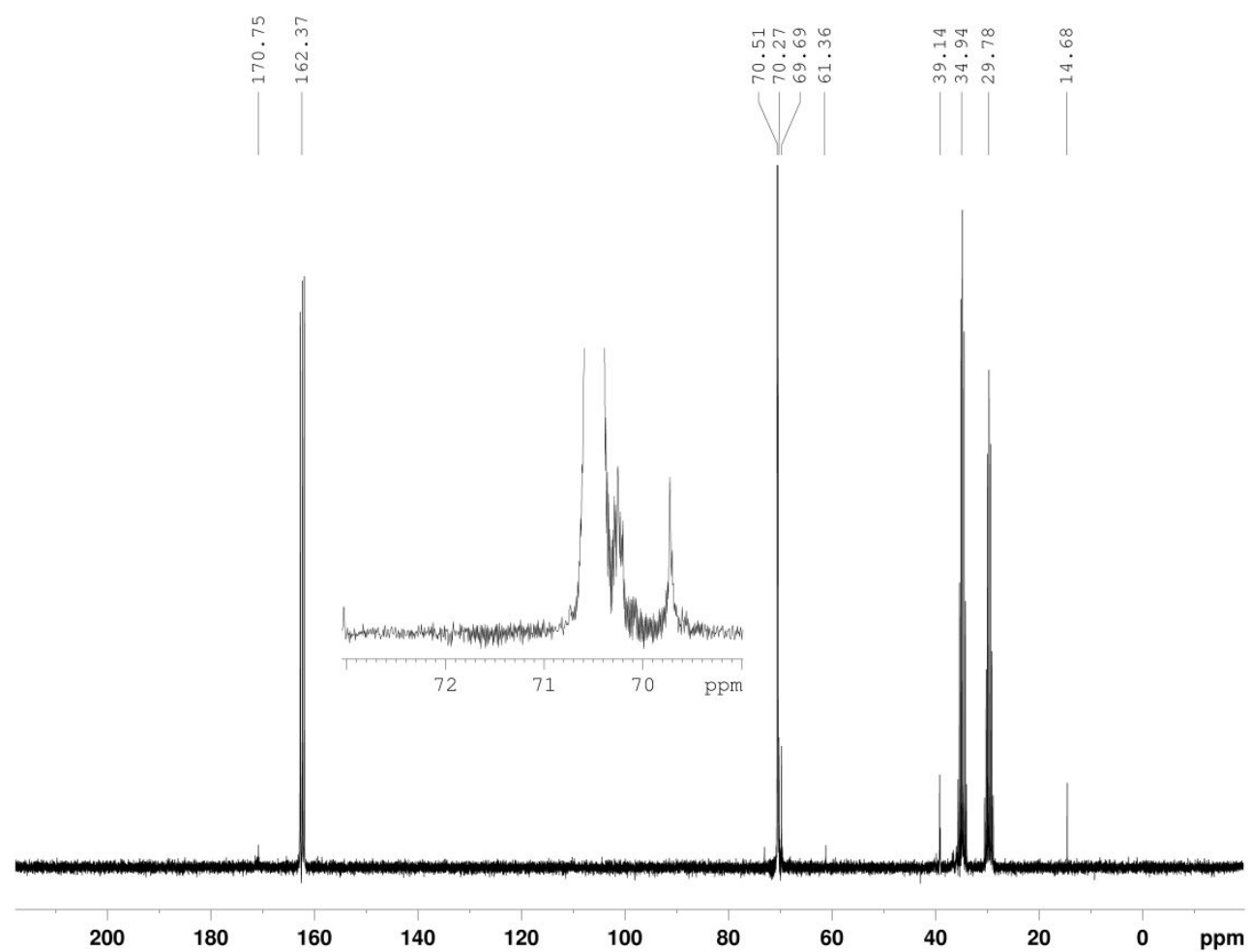

c)

Figure S5. ${ }^{1} \mathrm{H}$ NMR spectrum (300 MHz): $\mathrm{NH}_{2}$-PEG-CC (a) and CDs-PEG-CC (b). ${ }^{13} \mathrm{C}$ NMR of CDsPEGCC (c)

Rationale for the interpretation of the CDs and CDs-PEG-BT titration: de Levie approach.

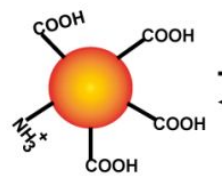

$\mathrm{L}^{+}$

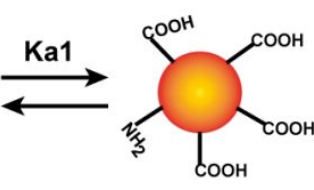

$\mathbf{L}^{0}$

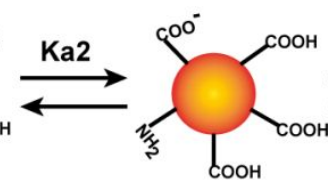

$\mathrm{L}^{-}$

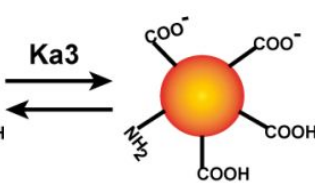

$\mathbf{L}^{2-}$

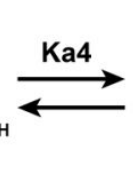

Scheme S1. approach

\section{Initial conditions:}

$V_{0}=$ initial solution volume

$c_{0}=$ initial CDs concentration

$c_{\mathrm{S}}=$ initial concentration of ionic strength stabilizer

$c_{\mathrm{B}}$ or $c_{\mathrm{A}}=$ strong base (in forward titration) or acid (in backward titration) titrant concentration $V_{\mathrm{B}}$ or $V_{\mathrm{A}}=$ Volume of the titrant added

$N=$ moles of strong acid possibly present as residual from the synthetic process or CDs pretreatments

(when negative, it accounts for moles of strong base) 


\section{Forward titration}

CDs mass balance:

$C_{C D s}=C_{L^{+}}+C_{L^{0}}+C_{L^{-}}+C_{L^{-2}}+C_{L^{-3}}+C_{L^{-4}}=\frac{C_{0} V_{0}}{V_{0}+V_{b}}(\mathbf{1})$

Equilibrium constants:

$K_{a 1}=\frac{C_{L^{0}} C_{H^{+}}}{C_{L^{+}}} \quad$ (2) $K_{a 2}=\frac{C_{L^{-}} C_{H^{+}}}{C_{L^{0}}} y^{2}$ (3) $K_{a 3}=\frac{C_{L^{2}} C_{H^{+}}}{C_{L^{-1}}} y^{4} \quad$ (4) $K_{a 4}=\frac{C_{L^{3}-} C_{H^{+}}}{C_{L^{2}}} y^{6}$

$K_{a 5}=\frac{C_{L^{4}}-C_{H^{+}}}{C_{L^{3-}}} y^{8}$ (6) $K_{W}=C_{O H^{-}} C_{H^{+}} y^{2}$

Solvent (water) dissociation equilibrium:

$K_{w}=H^{+} \mathrm{OH}^{-} y^{2}$

Concentration fractions

$\alpha_{0}=\frac{C_{L^{4-}}}{C}=\frac{K_{a 1} K_{a 2} K_{a 3} K_{a 4} K_{a 5}}{D}$

$\alpha_{2}=\frac{C_{L^{2-}}}{C}=C_{H^{+}}{ }^{2} y^{14} \frac{K_{a 1} K_{a 2} K_{a 3}}{D}$

$\alpha_{4}=\frac{C_{L^{0}}}{C}=C_{H^{+}}{ }^{4} y^{20} \frac{K_{a 1}}{D}$

$$
\begin{aligned}
& \alpha_{1}=\frac{C_{L^{3-}}}{C}=C_{H^{+}} y^{8} \frac{K_{a 1} K_{a 2} K_{a 3} K_{a 4}}{D} \\
& \alpha_{3}=\frac{C_{L^{-}}}{C}=C_{H^{+}}{ }^{3} y^{18} \frac{K_{a 1} K_{a 2}}{D} \\
& \alpha_{5}=\frac{C_{L^{+}}}{C}=\frac{C_{H^{+}}{ }^{5} y^{20}}{D}
\end{aligned}
$$

With

$D=C_{H^{+}}{ }^{5} y^{20}+C_{H^{+}}{ }^{4} y^{20} K_{a 1}+C_{H^{+}}{ }^{3} y^{18} K_{a 1} K_{a 2}+C_{H^{+}}{ }^{2} y^{14} K_{a 1} K_{a 2} K_{a 3}+C_{H}+y^{8} K_{a 1} K_{a 2} K_{a 3} K_{a 4}+K_{a 1} K_{a 2} K_{a 3} K_{a 4} K_{a 5}$

Ionic strength:

$I=\frac{1}{2}\left(C_{H^{+}}+C_{O H^{-}}+C_{C l^{-}}+C_{N a^{+}}+C_{L^{+}}+C_{L^{-}}+4 C_{L^{2-}}+9 C_{L^{3-}}+16 C_{L^{4-}}\right)$

where
$C_{O H^{-}}=\frac{K_{w}}{C_{H^{+}} y^{2}}$
$C_{N a^{+}}=\frac{C_{B} V_{B}+C_{S} V_{0}}{V_{B}+V_{0}}$
$C_{C l^{-}}=\frac{N+C_{S} V_{0}}{V_{0}+V_{B}}$
$C_{L^{+}}=\frac{\alpha_{5} C_{0} V_{0}}{V_{0}+V_{B}}$
$C_{L^{0}}=\frac{\alpha_{4} C_{0} V_{0}}{V_{0}+V_{B}}$
$C_{L^{-}}=\frac{\alpha_{3} C_{0} V_{0}}{V_{0}+V_{B}}$
$C_{L^{2-}}=\frac{\alpha_{2} C_{0} V_{0}}{V_{0}+V_{B}}$
$C_{L^{3-}}=\frac{\alpha_{1} C_{0} V_{0}}{V_{0}+V_{B}}$
(24) $C_{L^{4-}}=\frac{\alpha_{0} C_{0} V_{0}}{V_{0}+V_{B}}$ 
The activity coefficients (Davies equation):

$$
y=10^{-0.5\left[\frac{\sqrt{I}}{1+\sqrt{I}}^{-0.3 I}\right]}
$$

In this approximation the single ion activity coefficients can be expressed as

$$
y_{i}=y^{z^{2} i}(27)
$$

Charge balance:

$\left|\mathrm{H}^{+}\right|+\left|\mathrm{Na}^{+}\right|+\left|\mathrm{L}^{+}\right|=\left|\mathrm{Cl}^{-}\right|+\left|\mathrm{OH}^{-}\right|+\left|\mathrm{L}^{-}\right|+\left|2 \mathrm{~L}^{2-}\right|+\left|3 \mathrm{~L}^{3-}\right|+\left|4 \mathrm{~L}^{4-}\right|$

Combining the former equations, the following solving equation, representing the whole forward titration curve, can be obtained in terms of $V_{\mathrm{B}}$ as a function of $\mathrm{pH}$ :

$V_{B}=\frac{N+\left[C_{0}\left(4 \alpha_{0}+3 \alpha_{1}+2 \alpha_{2}+\alpha_{3}-\alpha_{5}\right)-\Delta\right] V_{0}}{\left(C_{B}+\Delta\right)}$

where:

$$
\Delta=\left|H^{+}\right|-\left|O H^{-}\right|=\frac{K_{w}}{\left|H^{+}\right| y^{2}}
$$

\section{Backward titration}

Mass balances, $K_{\mathrm{ai}}, K_{\mathrm{w}}$ and the $\alpha_{i}$ are the same as in the forward titration, while:

$$
\begin{aligned}
& I=\frac{1}{2}\left(C_{H^{+}}+C_{O H^{-}}+C_{C l^{-}}+C_{N a^{+}}+C_{L^{+}}+C_{L^{-}}+4 C_{L^{2-}}+9 C_{L^{3-}}+16 C_{L^{4-}} \quad\right. \text { (31) } \\
& C_{O H^{-}}=\frac{K_{w}}{C_{H^{+}} y^{2}}
\end{aligned}
$$

$C_{L^{+}}$

$$
=\frac{\alpha_{5} C_{0} V_{0}}{V_{B}+V_{0}+V_{A}}
$$

$$
C_{L^{0}}=\frac{\alpha_{4} C_{0} V_{0}}{V_{B}+V_{0}+V_{A}}
$$

$$
C_{L^{-}}=\frac{\alpha_{3} C_{0} V_{0}}{V_{B}+V_{0}+V_{A}}
$$

$$
C_{L^{2-}}=\frac{\alpha_{2} C_{0} V_{0}}{V_{B}+V_{0}+V_{A}}(\mathbf{3 8}) \quad C_{L^{3-}}=\frac{\alpha_{1} C_{0} V_{0}}{V_{B}+V_{0}+V_{A}}
$$

$$
C_{L^{4-}}=\frac{\alpha_{0} C_{0} V_{0}}{V_{B}+V_{0}+V_{A}}
$$

Combining all former conditions, the following solving equation, representing the whole backward titration curve, can be obtained in terms of $V_{B}$ as a function of $\mathrm{pH}$ : 
$V_{B}=\frac{N+\left[C_{0}\left(4 \alpha_{0}+3 \alpha_{1}+2 \alpha_{2}+\alpha_{3}-\alpha_{5}\right] V_{0}-\left(C_{B}+\Delta\right) V_{B}\right.}{\left(C_{A}+\Delta\right)}$

Table S1. ל-potential and drug loading capability of CDs and the parent compounds

\begin{tabular}{|l|c|c|}
\hline \multicolumn{1}{|c|}{ Sample } & $\begin{array}{c}\zeta \text {-potential } \\
(\mathbf{m V})\end{array}$ & $\begin{array}{c}\text { Drug Loading } \\
(\mathbf{w} / \mathbf{w} \%)\end{array}$ \\
\hline CDs & -22.0 & - \\
\hline CDs@IT & -12.3 & 6.9 \\
\hline CDs-PEG-CC & -12.6 & - \\
\hline CDs-PEG-CC@IT & -11.8 & 28.4 \\
\hline CDs-PEGBT & -5.58 & - \\
\hline CDs-PEGBT@IT & -5.93 & 16.1 \\
\hline
\end{tabular}
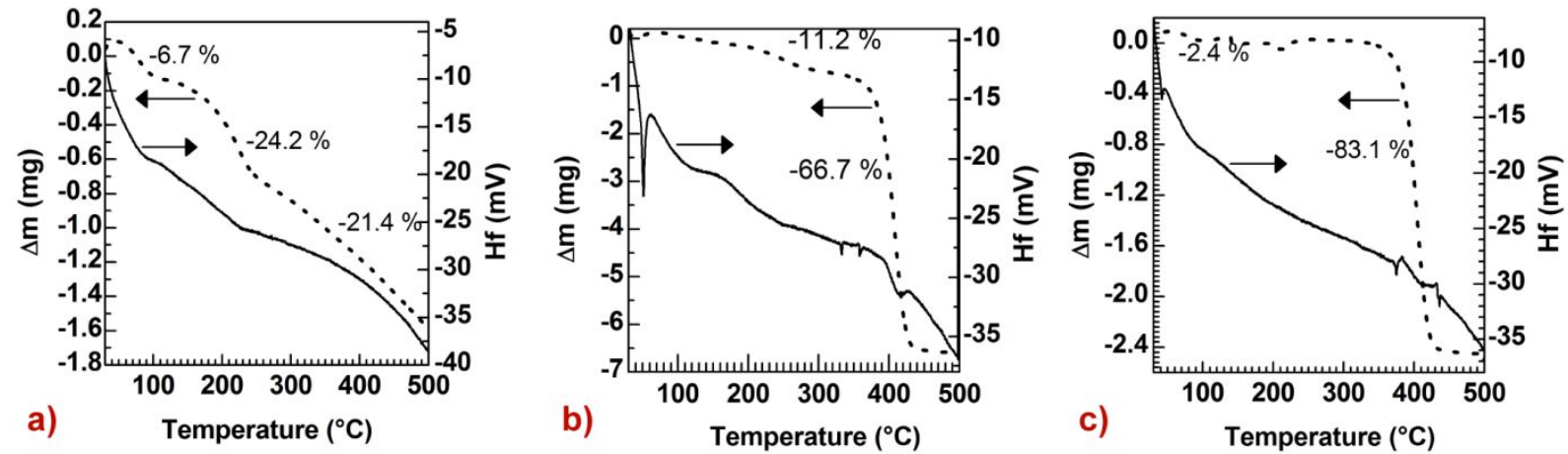

Figure S6. Differential Scanning Calorimetry (DSC) and Thermogravimetric Analysis (TGA) curves obtained for CDs (a), PEG-BT (b) and CDs-PEG-BT (c).

\section{In vitro cell viability study}


MDA-MB-231

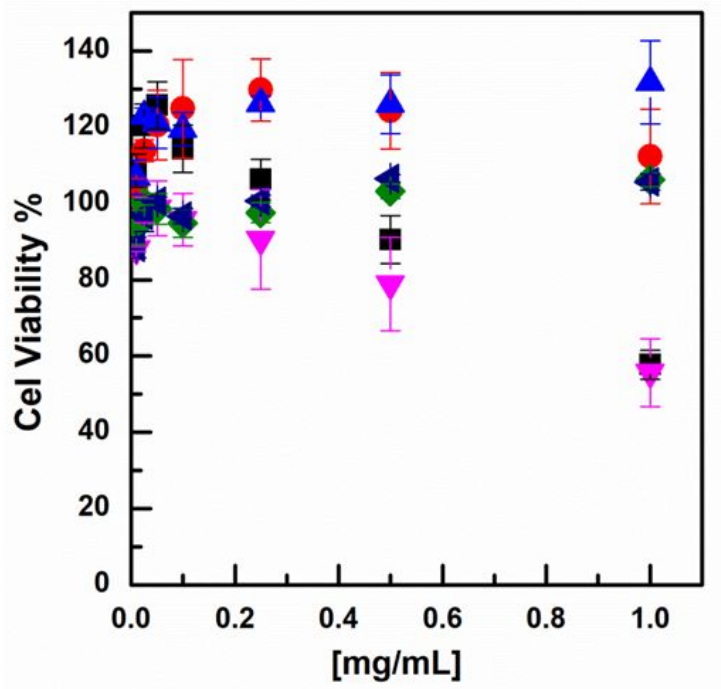

- CDs 24h

- CDs-PEG-CC 24h

$\triangle$ CDs-PEG-BT 24h
MCF7

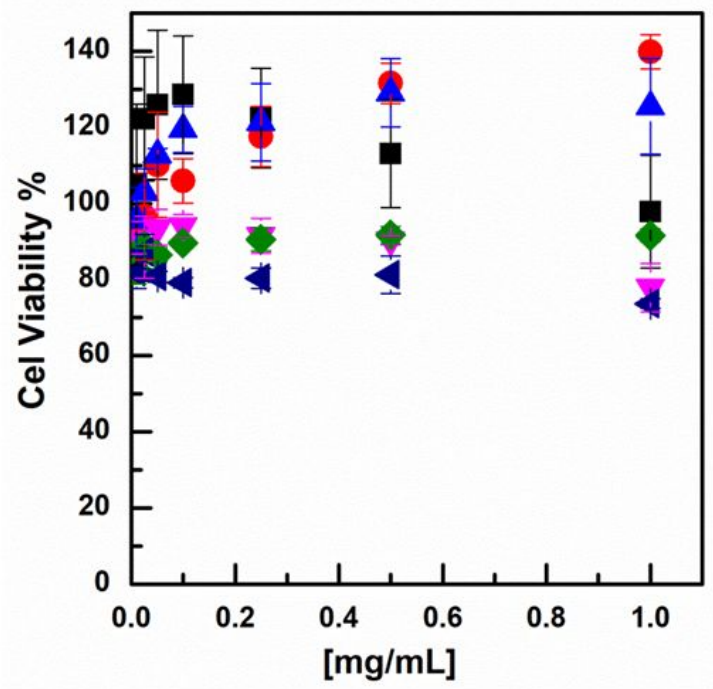

$\nabla$ CDs 48h

- CDs-PEG-CC 48h

4 CDs-PEG-BT 48h

Figure S7. Cell viability on 2-D human breast cancer cell cultures: MTS assay on MDA-MB-231 and MCF7 for CDs, CDs-PEG-CC and CDs-PEG-BT after 24 and 48h of incubation

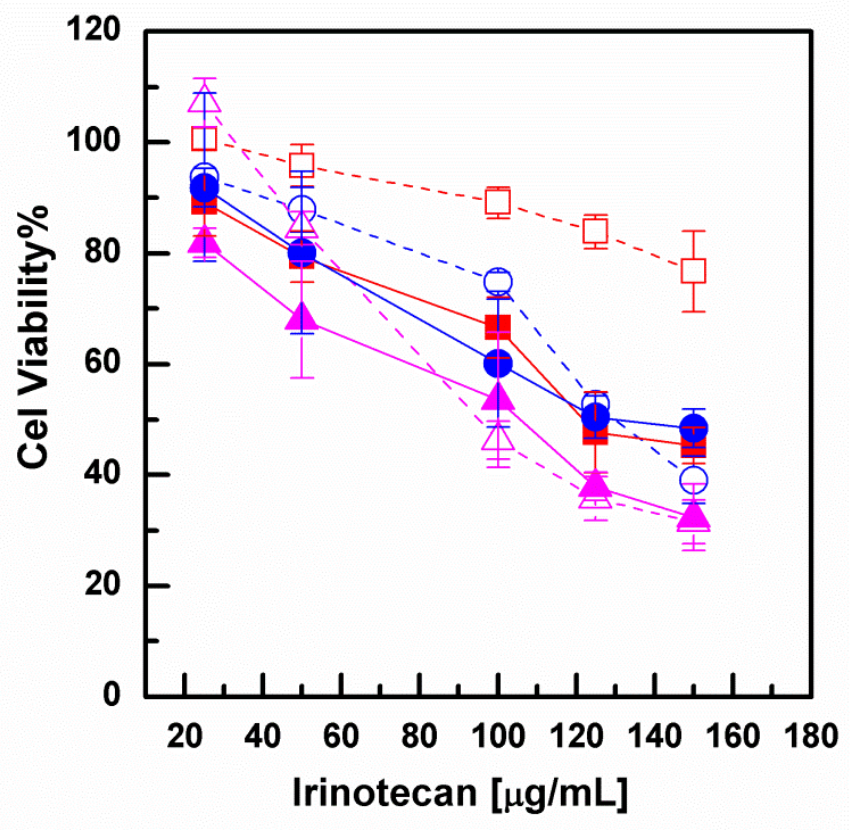


Figure S8. Anticancer activity on 2-D human breast cancer cell cultures (a): MTS assay on MCF7 (dashed lines) and MDA-MB-231 (solid lines) for irinotecan (purple), CDs-PEG-CC@IT (red) and CDs-PEG-BT@IT (blue) after 24h of incubation. 

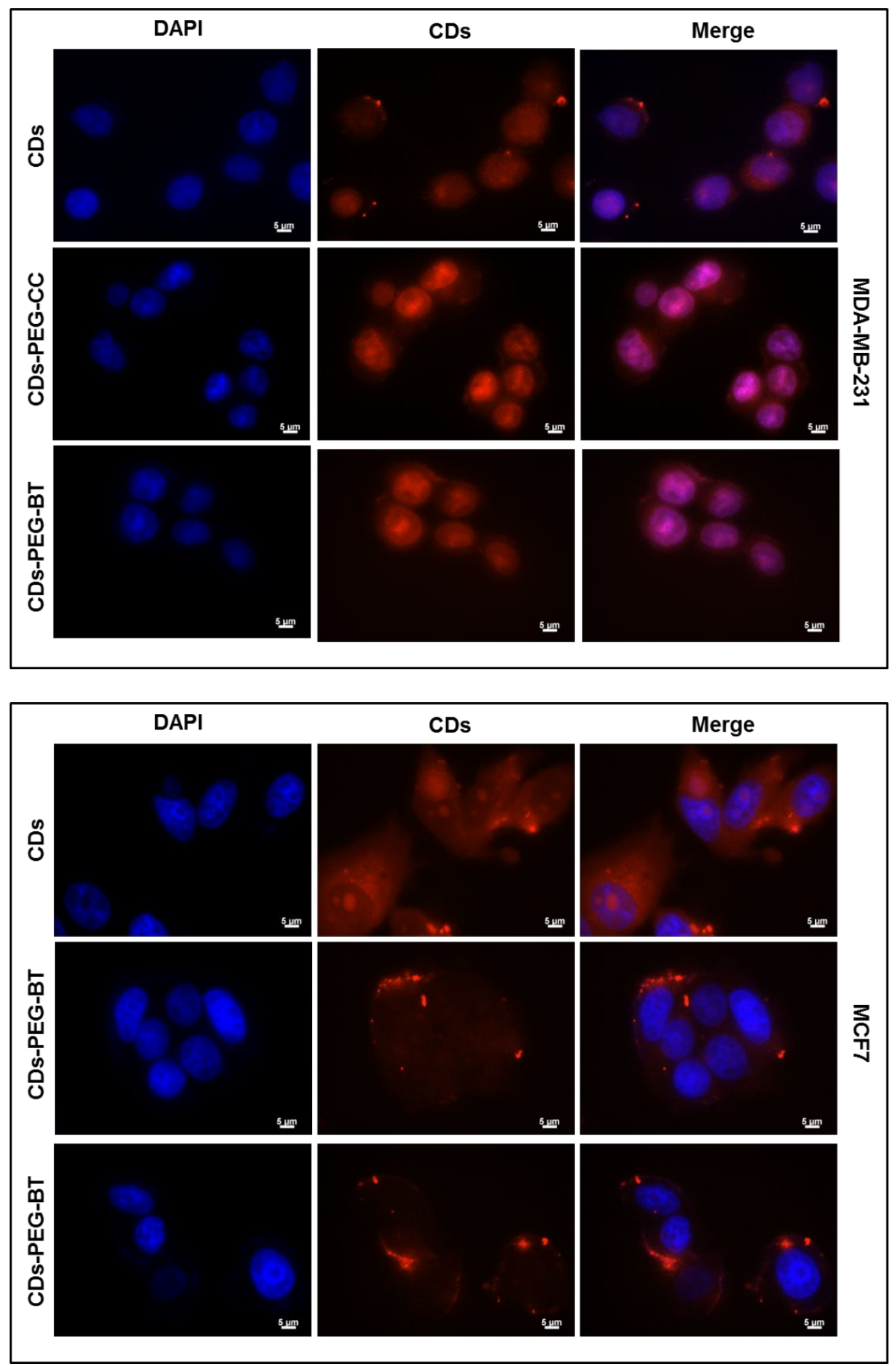

Figure S9. Uptake of CDs, CDs-PEG-CC and CDs-PEG-BT after 6h incubation on MDA-MB-231 and MCF7.

\section{Experimental Section}


Materials: 4-Pentynoic acid, N-Hydroxysuccinimide (NHS), N-(3-Dimethylaminopropyl)-N'ethylcarbodiimide hydrochloride (EDC HCl), Poly(ethylene glycol) bis(amine) 2,000 Da $\left(\mathrm{NH}_{2}-\mathrm{PEG}^{-}\right.$ $\mathrm{NH}_{2}$ ) were purchased from Sigma Aldrich (Italy).

Human breast cancer cells (MCF7 and MDA-MB-231) (purchased from Sigma Aldrich, Italy) were cultured in Dulbecco's Minimum Essential Medium (DMEM), supplemented with 10\% fetal bovine serum (FBS, Euroclone), 1\% of penicillin/streptomycin (10000 $\mathrm{U} \mathrm{mL}^{-1}$ penicillin and $10 \mathrm{mg} \mathrm{mL}^{-1}$ streptomycin, Euroclone) and $1 \%$ of L-glutamine (Euroclone), at $37{ }^{\circ} \mathrm{C}$ in $5 \% \mathrm{CO}_{2}$ humidified atmosphere. Cell Titer 96 Aqueous One Solution Cell Proliferation assay (MTS solution) were purchased from Promega.

Synthesis of PEG-CC: To synthesize PEG-CC, $\mathrm{NH}_{2}-\mathrm{PEG}-\mathrm{NH}_{2}(500 \mathrm{mg})$ was dissolved in phosphate buffer solution (PBS) $(8 \mathrm{ml})$ and the $\mathrm{pH}$ of this solution was measured as 10. Then, $29.4 \mathrm{mg}$ of 4pentynoic acid $(0.3 \mathrm{mmol})$ were added and after its complete dissolution the $\mathrm{pH}$ of mixture was adjusted to 6.4 , adding $\mathrm{HCl} 1 \mathrm{~N}$. Finally, $34.8 \mathrm{mg}$ of $\mathrm{NHS}(0.3 \mathrm{mmol})$ and $57.6 \mathrm{mg}$ of $\mathrm{EDC} \cdot \mathrm{HCl}(0.3$ mmol) were added and the reaction mixture $\mathrm{pH}$ value was maintained at 6.4 with $\mathrm{NaOH} 0.33 \mathrm{~N}$. The mixture was left under stirring to react at room temperature overnight. After the reaction time the $\mathrm{pH}$ of the reaction mixture was adjusted to 9.5 with $\mathrm{NaOH} 0.1 \mathrm{M}$ and then PEG-CC was purified by $\mathrm{d}$ by gel permeation chromatography using Sephadex G-15 as separating gel. After purification the PEG-CC solution was freeze dried from water; the pure product obtained was $87 \%$ yield. The obtained PEG derivative was characterized by ${ }^{1} \mathrm{H}$ NMR $\left(300 \mathrm{MHz}, \mathrm{D}_{2} \mathrm{O}\right)$.

Differential Scanning Calorimetry (DSC) and Thermogravimetric Analysis (TGA): The covalent surface functionalization of the CDs and the amount of PEG-BT chains were assessed by DSC and TGA analysis, using a DSC/TGA 131 EVO (by SETARAM Instr.). The measures were performed under nitrogen flow $\left(1 \mathrm{~mL} \mathrm{~min}^{-1}\right)$ using about $3 \mathrm{mg}$ of dried sample placed into an aluminum crucible. The heating rate was: $30-500{ }^{\circ} \mathrm{C}, 10^{\circ} \mathrm{C} \mathrm{min}^{-1}$. 
In vitro cell viability study: The cell viability of $\mathrm{CDs}$, CDs-PEG-CC and CDs-PEG-BT was assessed by the MTS assay on human breast cancer cell lines (MCF-7, MDA-MB-231). Cells were seeded in a 96-multiwell plate at a density of $1 \times 10^{4}$ cells/well and grown in supplemented DMEM. After $24 \mathrm{~h}$, the medium was replaced with $200 \mu \mathrm{L}$ of fresh culture medium containing CDs and CDs-PEG-CC at concentration per well ranging from 0.01 to $1.4 \mathrm{mg} \mathrm{mL}^{-1}$. Untreated cells were used as negative control. After 24 and $48 \mathrm{~h}$, samples were taken away from the wells and substituted by fresh medium (100 $\mu \mathrm{L})$ and $20 \mu \mathrm{L}$ of a MTS solution. Cells were incubated for additional $2 \mathrm{~h}$ at $37^{\circ} \mathrm{C}$, and then the absorbance at $492 \mathrm{~nm}$ was measured using a microplate reader (Multiskan, Thermo, U.K.). Results were expressed as percentage reduction of the control cells. All culture experiments were performed in triplicates. In another experimental set the cytotoxicity of CDs-PEG-CC@IT and CDs-PEG-BT@IT on MCF7 and MDA-MB-231 was evaluated after $24 \mathrm{~h}$ of incubation. Briefly, cells were incubated with CDs-PEGCC@IT, CDs-PEG-BT@IT and irinotecan as positive control at drug concentration per well equal to $25,50,100,150,125$ and $150 \mu \mathrm{g} \mathrm{mL}-1$ and after $24 \mathrm{~h} \mathrm{MTS}$ assay was performed as above reported.

2D-Cell uptake: The cellular uptake of CDs and empty CDs-PEG-CC was evaluated by fluorescent microscopy (Zeiss “AXIO Vert. A1” Microscope Inverted). In particular, MCF-7 and MDA-MB-231 cells were seeded at a density of $3 \times 10^{4}$ cells/well into 8 well plate and cultured for $24 \mathrm{~h}$. Then the medium was replaced with $500 \mu \mathrm{L}$ of fresh DMEM containing $0.5 \mathrm{mg} \mathrm{mL}^{-1}$ of CDs or empty CDsPEG-CC and cells were incubated for $6 \mathrm{~h}$. Thus the medium was removed, the cell monolayer was washed twice with PBS pH 7.4 and the nuclei were stained with 4',6-diamidino-2-phenylindole (DAPI). Images were recorded by a fluorescence microscope using an Axio Cam MRm (Zeiss). Untreated cells were used as negative control to set the auto fluorescence. 